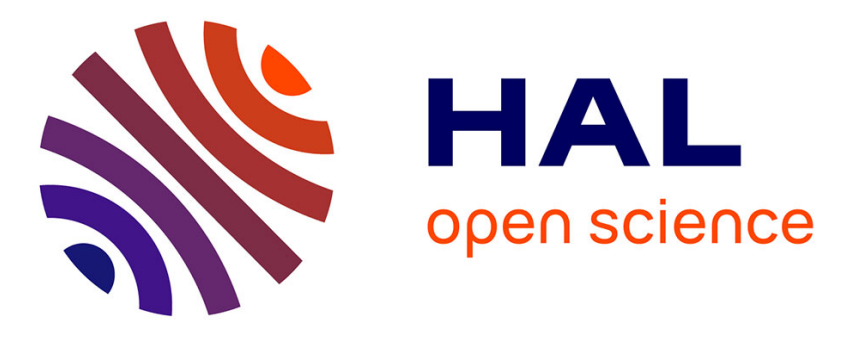

\title{
Smoking and FOS expression from blood leukocyte transcripts in patients with coronary artery disease.
}

Sebastien Saliques, Jean-Raymond Teyssier, Catherine Vergely, Luc Lorgis, Julie Lorin, Anne Donzel, Pierre Sicard, Juliane Berchoud, Sylviane Ragot, Claude Touzery, et al.

\section{To cite this version:}

Sebastien Saliques, Jean-Raymond Teyssier, Catherine Vergely, Luc Lorgis, Julie Lorin, et al.. Smoking and FOS expression from blood leukocyte transcripts in patients with coronary artery disease.. Atherosclerosis, 2011, 219 (2), pp.931-6. 10.1016/j.atherosclerosis.2011.09.026 . inserm-00828274

\section{HAL Id: inserm-00828274 https://www.hal.inserm.fr/inserm-00828274}

Submitted on 30 May 2013

HAL is a multi-disciplinary open access archive for the deposit and dissemination of scientific research documents, whether they are published or not. The documents may come from teaching and research institutions in France or abroad, or from public or private research centers.
L'archive ouverte pluridisciplinaire HAL, est destinée au dépôt et à la diffusion de documents scientifiques de niveau recherche, publiés ou non, émanant des établissements d'enseignement et de recherche français ou étrangers, des laboratoires publics ou privés. 


\title{
Smoking and FOS expression from blood leukocyte transcripts in patients with coronary artery disease
}

\author{
Sebastien Saliques ${ }^{\mathrm{a}}$, Jean-Raymond Teyssier ${ }^{\mathrm{b}}$, Catherine Vergely ${ }^{\mathrm{a}}$, Luc Lorgis ${ }^{\mathrm{a}, \mathrm{c}}$, Julie Lorin ${ }^{\mathrm{a}}$, \\ Anne Donzel $^{\mathrm{b}}$, Pierre Sicard ${ }^{\mathrm{a}}$, Juliane Berchoud ${ }^{\mathrm{c}}$, Sylviane Ragot ${ }^{\mathrm{b}}$, Claude Touzery ${ }^{\mathrm{c}}$, Yves Cottin ${ }^{\mathrm{a}, \mathrm{c}}$, \\ Luc Rochette $^{\mathrm{a}}$, Marianne Zeller ${ }^{\mathrm{a}, *}$ \\ a IFR 100 Santé-STIC, Laboratoire de Physiopathologie et Pharmacologie Cardiovasculaires Expérimentales, UFR Médecine, Université de Bourgogne (EA2979), France \\ ${ }^{\mathrm{b}}$ Laboratoire de Génétique Moléculaire, CHU Dijon, France \\ ' Service de Cardiologie, CHU Dijon, France
}

\section{A R T I C L E I N F O}

\section{Article history:}

Received 15 June 2011

Received in revised form 12 August 2011

Accepted 11 September 2011

Available online 21 September 2011

\section{Keywords:}

Smoking

Blood leukocyte transcriptome

c-Fos

Coronary artery disease

\begin{abstract}
A B S T R A C T
Objective: Analysis of the leukocyte transriptome, in particular the Finkel-Biskis-Jinkins Osteosarcoma (c-Fos) gene, which has a prominent role in inflammation, provides new insights into atherosclerosis mechanisms. Although smoking is a major risk factor, the links between smoking status and coronary artery disease (CAD) remains unclear. We aimed to analyze the relationship between smoking status and c-Fos expression in circulating leukocytes of patients with CAD.

Methods: c-Fos expression was measured by RT-Q-PCR, from blood leukocytes of 239 consecutive patients after acute myocardial infarction (MI). The patients were asked about their smoking status and stratified into 3 groups: current smokers (CS) $(N=85)$, past smokers (PS) $(N=78)$ and never smokers $(N S)(N=76)$. Results: NS had a higher risk profile including hypertension, and CS were younger than PS and NS ( -13 years and 17 years respectively). There was only a trend towards lower CRP levels in NS and PS than in CS. The mean $c$-Fos transcript level was slightly higher in CS than in PS and NS (0.924 vs. 0.908 and $0.861 \mathrm{AU}$, respectively; $p=0.005$ ). By univariate analysis, neither age, nor sex, nor CRP nor white blood cell count was associated with $c$-Fos transcript levels. By multivariate analysis, CS (vs. PS + NS) was the strongest predictor of the $c$-Fos transcript level, $(B=0.042 \pm 0.014, p=0.003)$, even after adjustment for confounding factors (i.e. hypertension, chronic medication, family history of CAD, and prior MI).

Conclusion: Our work suggests that c-Fos transcript level in blood leukocyte could be considered a cumulative biomarker of smoking. As the $c$-Fos gene has been put forward as a new factor in the progression and severity of atherosclerosis, it could be considered a novel potential pathway of tobacco toxicity in coronary artery disease.
\end{abstract}

(c) 2011 Elsevier Ireland Ltd. All rights reserved.

\section{Introduction}

Smoking is a major avoidable cause of cardiovascular disease worldwide [1] and was responsible for around 100 million deaths during the 20th century. This could rise to 1 billion deaths during the 21st century [2]. Smoking is associated with circulatory and vascular alterations, including atherosclerosis [3], endothelial dysfunction [4], altered blood lipid profile [5], increased platelet aggregation [6] and oxidative stress-associated biomarkers of inflammation [7]. However, the mechanisms by which smoking

\footnotetext{
* Corresponding author at: Laboratoire de Physiopathologie et Pharmacologie Cardiovasculaires Expérimentales, UFR Médecine, 7Bd Jeanne d'Arc, 21000 Dijon, France. Tel.: +33 380393292; fax: +33 380393293.

E-mail address: marianne.zeller@u-bourgogne.fr (M. Zeller).
}

influences atherosclerosis, and in particular coronary artery disease (CAD), are still not completely understood.

Finkel-Biskis-Jinkins Osteosarcoma (c-Fos) stress-induced transcription factors, which respond to and integrate many signaling pathways, are involved in inflammation and pathogenic changes in atherosclerosis [8]. c-Fos has been shown to be strongly expressed in the leukocytes of patients with atherosclerosis and has been described as a better marker of atherosclerosis than CRP [8]. Moreover, circulating $c$-Fos transcripts have also been shown to provide additional insights into the biology of cardiovascular (CV) diseases, suggesting that $c$-Fos expression in the blood could serve as a sensitive marker to improve treatment outcome [9]. However, there are very few data on the impact of smoking on this key oncogene. Comprehensive gene expression profiles reveal that c-Fos is specifically expressed in the lung tissue of smokers [10]. In adult rat aortic VSMC and adventitial fibroblasts, exposure to nicotine, a major 
component of cigarette smoke, exerts growth-promoting effects on vascular cells through the enhancement of angiotensin-induced DNA synthesis and c-Fos expression [11].

In a prospective study, we aimed to analyze the relationship between smoking status and c-Fos mRNA expression in the blood leukocytes of patients with CAD.

\section{Methods}

\subsection{Patients}

All consecutive patients aged $>18$ years and hospitalized $<24 \mathrm{~h}$ after symptoms onset for acute myocardial infarction (MI) in the coronary care unit of Dijon University Hospital between 1st March and 31st July 2009 were included. Patients with malignancy were excluded from the study. MI was defined by an increase in serum troponin I ( $2 \times$ upper limit of the hospital normal range) associated with symptoms of ischemia and/or characteristic electrocardiographic (ECG) signs. The present study complied with the Declaration of Helsinki and was approved by the ethics committee of University Hospital of Dijon. Each patient gave written consent before participation.

\subsection{Data collection}

For each patient, data on demographics, risk factors (history of hypertension, diabetes, hyperlipidemia, obesity \{defined as a body mass index $(\mathrm{BMI}) \geq 30\})$, a family history of CAD [i.e. parental history of premature $(<55$ years for men and $<65$ years for women) CAD in first degree relative] and prior MI were prospectively collected. Clinical data, including hemodynamic parameters on admission, left ventricular ejection fraction (LVEF) and location of infarction were recorded. LVEF was determined by echocardiography at $2 \pm 1$ days after admission.

\subsection{Angiographic data}

The coronary angiography was performed via the femoral or radial approach. The number of diseased vessels (stenosis $>50 \%$ ) was determined. The angiograms were analyzed by 2 expert investigators blinded to the clinical and biological data. Among the study population $(n=239), 15(6 \%)$ patients did not undergo coronary angiography.

\subsection{Biological data}

Blood samples were drawn at admission [time from symptom onset to blood sampling: $240(160-579) \mathrm{min}$ ] to assess biological variables.

Homocysteine concentrations were determined by chemiluminescence on an Immulite 2000 analyzer (Diagnostic Products Corporation, Los Angeles, USA), C-reactive protein (CRP), total cholesterol (TC), high-density lipoprotein cholesterol (HDL-C) and triglyceride (TG) concentrations were measured on a Dimension analyzer (Dade Behring, Newark, NE) using immunonephelometry assay. Low-density lipoprotein cholesterol (LDL-C) levels were calculated using the Friedewald formula [12]. Plasma glucose concentrations [enzymatic method (glucose oxidase)] and creatinine levels were measured on a Vitros 950 analyzer (Ortho Clinical Diagnostics, Rochester, NY). The MDRD (Modification of the Diet in Renal Disease) level was calculated as $186.3 \times(\mathrm{Cr})-1.154 \times($ age $)-0.203 \times(0.742$ if female $) \times(1.21$ if African American) [13]. Glycated hemoglobin A1 C (HbA1c) was measured with ion exchange HPLC (Bio-Rad Laboratories, Richmond, CA). Creatine kinase (CK) peaks were assessed by sampling every $8 \mathrm{~h}$ during the first two days after admission (Dimension Vista
Intelligent Lab System, Siemens). Plasma N-terminal pro B-type natriuretic peptide (NT-proBNP) was determined by ELISA with an Elecsys NT-proBNP sandwich immunoassay on Elecsys 2010 (Roche Diagnostics, Basel, Switzerland). Peak creatine kinase (CK) was assessed by sampling every $8 \mathrm{~h}$ during the first two days after admission (Dimension Vista Intelligent Lab System, Siemens).

\section{5. $m R N A$ extraction and Quantitative RT-PCR}

Blood samples were salted-out in a lysing solution (Tris HCL pH 7.5; $50 \mathrm{mM} ; \mathrm{MgCl} 225 \mathrm{mM} ; \mathrm{NaCl} 50 \mathrm{mM}$ ) to obtain a total leukocyte pellet. mRNA were obtained using a TRIZOL ${ }^{\circledR}$ (Invitrogen, Paisley, UK) extraction assay protocol on the leukocyte pellet, and reverse transcribed using Murine Moloney Leukemia Virus Reverse Transcriptase (Invitrogen, Paisley, UK).

The levels of the transcripts were quantified by the relative gene expression analysis method using Q-PCR technique, in duplicate at least two or three times. Briefly, for each amplification, aliquots of cDNA from 50 ng of total RNA were amplified by the Q-PCR technique on the Rotorgene 3000 (Corbett Research, Cambridge, UK), using the Quantifast SYBR Green PCR kit (Qiagen S.A., France) in a $25 \mu \mathrm{L}$ reaction volume. PCR amplification was run on a Light Cycler 480 (Roche Diagnostics, France). The GAPDH gene was selected as the reference (normalizing) gene after testing the homogenous expression of three genes (GAPDH, B2M and ACTB) using geNorm software. Each gene sequence was amplified in triplicate for 45 cycles under standardized conditions and the results were expressed as the mean of the three Cycle Threshold $(\mathrm{Ct})$ values. The reaction mixture contained $200 \mathrm{ng}$ of each primer and the PCR cycle parameters were: $94^{\circ}$ for $15 \mathrm{~s}, 60^{\circ}$ for $1 \mathrm{~min}, 72^{\circ}$ for $40 \mathrm{~s}$. The sequences of the primers selected by the Primer3 software (Whitehead Institute for Biomedical Research) were tested for their specificity by obtaining the melting curves at the end of each PCR. The $c$-Fos specific primers were: forward 5'-GGA GGA CCT TAT CTG TGC GTG A-3', and reverse 5'-GAA CAC ACT ATT GCC AGG AAC ACA-3'. The GAPDH specific primers were: forward 5'-CAT CTC TGC CCC CTC TGC T-3', and reverse $5^{\prime}$-ACG CCT GCT TCA CCA CCT $\mathrm{T}-3^{\prime}$. The PCR efficiency was established at 0.95 by the standard curve. RT-PCR was normalized by measuring the average $\mathrm{Ct}$ ratio in the investigated genes and in the control gene, glyceraldehyde3-phosphate dehydrogenase (GAPDH).

\subsection{Group definition}

Patients were rigorously asked about their self-reported smoking status by structured interviews conducted at the time of their hospital admission. Current smokers and patients who had stopped smoking $<3$ months earlier were classified as current smokers. Patients who had stopped smoking $\geq 3$ months earlier were classified as past smokers and those who had never smoked were classified as never smokers.

\subsection{Statistical analysis}

Continuous variables are presented as medians (25-75th percentile) or means $\pm S D$, as appropriate or as proportions. For continuous variables, a Kolmogorov-Smirnov analysis was performed to test normality. One Way ANOVA was performed to compare the 3 groups (current, past and never smokers). Dichotomous variables, expressed as numbers (\%), were compared by Chi-square tests for the 3 groups. For the comparison between 2 groups (univariate predictor of c-FOS level, Table 2), Pearson's or Spearman's rank correlation was used for continuous variables, and Student $t$ test or Wilcoxon test was used for dichotomous variables. Multiple linear regression analysis was performed with the c-Fos transcript level as a dependent variable, to test the indepen- 


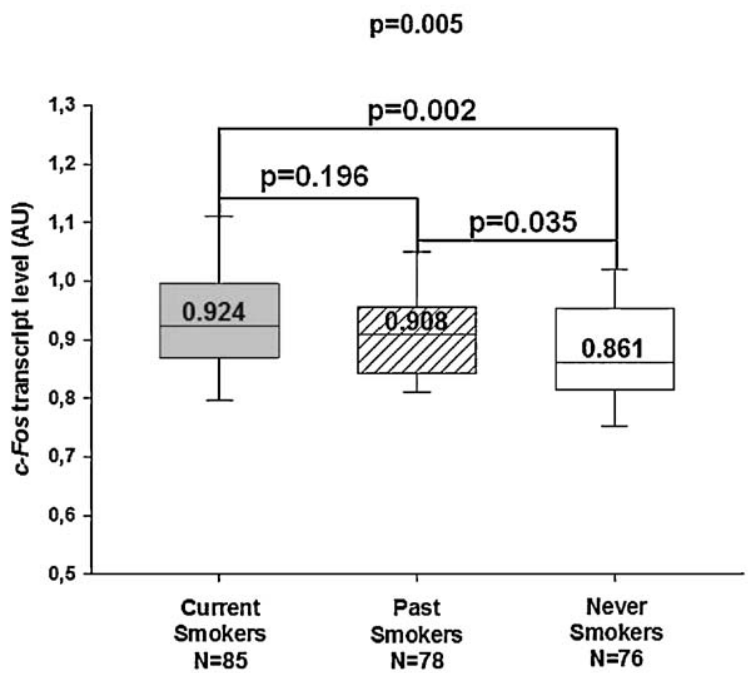

Fig. 1. c-Fos transcript level according to smoking status (AU). Patients who currently smoke or had stopped smoking $<3$ months were classified as current smokers $(n=85)$. Patients who had stopped cigarette consumption $\geq 3$ months were classified as past smokers $(n=78)$ and those who had never smoked were classified as never smokers $(n=76)$

dent predictors of FOS expression. Variables entered into the model were those with a significant relationship $(p<0.05)$ in univariate analysis. Given that only CS, but not PS or NS, is expected to be associated with the risk of MI, we focused our multivariate analysis on CS for more clinical relevance. Smoking was then entered as a dichotomous variable (current smoking vs. past or never smokers). In accordance with the Bonferroni correction, the alpha value was lowered to 0.02 to account for the number of comparisons being performed.

\section{Results}

\subsection{Characteristics of the study population}

During the inclusion period, 239 patients were included in the study. Baseline characteristics of the population according the smoking status are shown in Table 1 . As expected, never smokers were the oldest and current smokers the youngest.

Furthermore, never smokers had a higher risk profile, including hypertension and hypercholesterolemia. CS were younger than PS and NS ( -13 years and 17 years respectively) and more likely to be male. Cardiovascular drugs, except aspirin, were more frequently used in the PS and NS groups. Blood glucose levels were lower for CS. Kidney function as assessed by MDRD gradually deteriorated from current to never smokers, as age increased. CRP levels was similar for the 3 groups.

One major finding of our study was that the mean c-Fos transcript level in CS was markedly higher than that in PS and the difference was even greater for NS (0.924 [0.869-0.997] AU vs. 0.908 [0.841-0.955] AU and 0.861 [0.817-0.958] AU, respectively, $p=0.005$ ) (CS vs. PS: $p=0.196$; PS vs. NS: $p=0.035$; CS vs. NS: $p=0.002$ ) (Fig. 1).

\subsection{Determinants of c-Fos transcript level}

All of the parameters listed in Table 1 were tested for their relationship with the $c$-Fos transcript level, and the results are presented in Table 2 . The $c$-Fos transcript level gradually increased with HbA1c and homocysteine levels $(p=0.125$ and $p=0.168$ respectively). Neither blood lipids (LDL-cholesterol, triglyceride, HDL-cholesterol or total cholesterol), nor systemic inflammation markers (leukocyte count or CRP) were significantly associated with the $c$-Fos transcript level.

In contrast, $c$-Fos transcript levels tended to be higher in hypertensive patients $(p=0.188)$. Interestingly, patients with a family history of CAD or prior MI had a lower $c$-Fos transcript level $(p=0.070$ and $p=0.064$, respectively). No clinical parameter was significantly associated with the biomarker. The $c$-Fos transcript level was slightly lower in patients under ACE inhibitors $(p=0.174)$ or beta blockers $(p=0.067)$, which could explain the lower level of $c$-Fos in patients with prior MI. The frequency of diabetes in patients with prior MI was similar to that in patients without prior MI (9(31\%) vs. 48(19\%), $p=0.110)$. Moreover, the levels of homocysteine and $\mathrm{HbA1c}$ were similar in patients with and without prior $\mathrm{MI}(14.6 \pm 6.8 \mu \mathrm{mol} / \mathrm{L}$ vs. $13.6 \pm 6.3 \mu \mathrm{mol} / \mathrm{L}, p=0.440$ and $6.2 \pm 1.1$ vs. $6.1 \pm 1.2, p=0.572$, respectively).

We further performed linear regression analyses to test the predictors of $c$-Fos transcript levels. Four variables showed a significant association with $c$-Fos transcript levels by univariate analysis $(p<0.05)$ (i.e. current smoking, family history of CAD, prior MI, treatment with ACE inhibitors or beta blockers. In order to avoid multicollinearity between prior MI and chronic treatment with ACE inhibitors or beta blockers, only prior MI was introduced into the model. The multivariate model showed that only smoking status and a family history of CAD remained independent predictors of the $c$-Fos transcript level $(\beta=0.042, \mathrm{SE}=0.014, p=0.003$ and $\beta=-0.036, \mathrm{SE}=0.015, p=0.016$, respectively; $R^{2}=0.075$ ). Current smoking was the strongest predictor of elevated levels of $c$-Fos transcripts $(p=0.003)$. Given the major difference in risk factors among the 3 smoking status groups, in particular for age, gender, hypertension, diabetes and hypercholesterolemia, we performed a series of additional analyses by including each of these variables in the multivariate linear regression model. These risk factors were included as forced individual variables, with prior MI, a familial history of CAD and current smoking as covariates. Neither age $(p=0.202)$, female gender $(p=0.697)$, hypertension $(p=0.292)$, diabetes $(p=0.862)$ nor hypercholesterolemia $(p=0.397)$ were significantly associated with FOS. These additional analyses did not alter the strength of the predictive value of smoking on the $c$ Fos transcript levels. Patients on chronic therapy with ACEi or Betablockers showed a trend towards lower levels of FOS ( $p=0.174$ and $p=0.067$, respectively (Table 2 )). Treatments such as ACEi or betablockers may therefore have affected FOS transcript levels. In a comprehensive univariate linear regression analysis, we tested treatments with ACEi or betablockers for their relationship with FOS levels. The use of betablockers or ACEi was associated with only a trend towards lower levels of FOS $(\beta=-0.032, \mathrm{SE}=0.017$, $p=0.057 ; \beta=-0.017, \mathrm{SE}=0.017, p=0.310)$. Only the use of the combined variable [betablocker or ACEi] was significantly associated with lower FOS transcript levels $(\beta=-0.032, \mathrm{SE}=0.015, p=0.027$ ). When included in the multivariate model including familial history and smoking, this combined variable was not independently associated with FOS transcript levels $(\beta=-0.025, \mathrm{SE}=0.015, p=0.089$ ). Prior MI was not included in this model to avoid multicollinearity.

\section{Discussion}

Our prospective study in patients with CAD shows for the first time a strong association between c-Fos gene transcript levels in blood leukocytes and smoking. Our data further suggest that this association is strikingly independent of classical inflammation markers such as CRP or white blood cells (WBC). c-Fos is a transcription factor involved in many signaling pathways, and given its prominent role in atherosclerosis severity, our study suggests that it may be implicated in a novel pathophysiological mechanism that underlies the biological effects of smoking. 
Table 1

Patients characteristics. $n(\%)$, mean \pm SD or median (IQR).

\begin{tabular}{|c|c|c|c|c|}
\hline & Current smokers, $N=85$ & Past smokers, $N=78$ & Never smokers, $N=76$ & $p$ \\
\hline \multicolumn{5}{|l|}{ Risk factors } \\
\hline Age, years & $55 \pm 10$ & $68 \pm 13$ & $72 \pm 12$ & $<0.001$ \\
\hline Female & $13(20)$ & $6(8)$ & $40(52)$ & $<0.001$ \\
\hline Hypertension & $27(32)$ & $42(54)$ & $48(63)$ & $<0.001$ \\
\hline Obesity & $16(19)$ & $16(21)$ & $19(26)$ & 0.619 \\
\hline Diabetes & $15(18)$ & $11(14)$ & $22(29)$ & 0.056 \\
\hline Hypercholesterolemia & $29(34)$ & $39(50)$ & $36(47)$ & 0.089 \\
\hline Family history of CAD & $28(33)$ & $20(26)$ & $21(34)$ & 0.566 \\
\hline Prior MI & $6(7)$ & $10(13)$ & $8(11)$ & 0.467 \\
\hline \multicolumn{5}{|l|}{ Clinical data } \\
\hline LVEF, \% & $54 \pm 12$ & $54 \pm 12$ & $54 \pm 12$ & 0.128 \\
\hline Systolic blood pressure, mmHg & $138 \pm 30$ & $139 \pm 28$ & $141 \pm 31$ & 0.815 \\
\hline Diastolic blood pressure, $\mathrm{mmHg}$ & $82 \pm 19$ & $82 \pm 19$ & $78 \pm 17$ & 0.429 \\
\hline Heart rate, $\mathrm{b} / \mathrm{min}$ & $76 \pm 21$ & $76 \pm 15$ & $82 \pm 20$ & 0.053 \\
\hline Anterior wall location & $27(31 \%)$ & $27(35 \%)$ & $31(42 \%)$ & 0.349 \\
\hline \multicolumn{5}{|l|}{ Chronic treatments } \\
\hline ACE inhibitor & $11(12)$ & $21(27)$ & $21(28)$ & 0.033 \\
\hline Statin & $13(15)$ & $26(33)$ & $23(30)$ & 0.017 \\
\hline Aspirin & $15(18)$ & 15(19) & 13(17) & 0.955 \\
\hline Betablocker & $10(12)$ & $18(23)$ & $26(34)$ & 0.002 \\
\hline Fibrate & $3(4)$ & $0(0)$ & $6(8)$ & 0.032 \\
\hline Oral antidiabetic drug & $8(9)$ & $8(10)$ & $15(20)$ & 0.086 \\
\hline \multicolumn{5}{|l|}{ Biological data } \\
\hline Time to sampling, min & $230(140-530)$ & 240(169-639) & $206(170-560)$ & 0.267 \\
\hline Glucose, $\mathrm{mmol} / \mathrm{L}$ & $6.5(5.5-8.0)$ & $7.2(6.0-8.5)$ & $7.2(6.2-10.0)$ & 0.017 \\
\hline HbA1c, \% & $5.7(5.5-6.1)$ & $5.7(5.5-6.2)$ & $5.8(5.5-6.3)$ & 0.394 \\
\hline MDRD, mL/min & $86.0(70.9-100.4)$ & $73.0(56.8-89.9)$ & $66.4(51.6-77.7)$ & $<0.001$ \\
\hline $\mathrm{CRP}, \mathrm{mg} / \mathrm{L}$ & $4.6(2.9-9.75)$ & $5.0(2.9-11.9)$ & $6.0(2.9-24.9)$ & 0.468 \\
\hline Homocysteine, $\mu \mathrm{mol} / \mathrm{L}$ & $12(9-18)$ & $12(9-15)$ & $12(9-16)$ & 0.421 \\
\hline HDL-cholest, g/L & $0.43(0.32-0.49)$ & $0.42(0.36-0.50)$ & $0.45(0.38-0.53)$ & 0.129 \\
\hline Triglycerides, g/L & $1.40(0.93-2.11)$ & $1.35(0.89-1.87)$ & $1.15(0.80-1.80)$ & 0.120 \\
\hline LDL-cholest, g/L & $1.24(0.97-1.52)$ & $1.22(0.97-1.51)$ & $1.21(0.93-1.46)$ & 0.730 \\
\hline Total-cholest, g/L & $1.99 \pm 0.51$ & $1.97 \pm 0.45$ & $1.97 \pm 0.52$ & 0.978 \\
\hline Leukocyte count, $10^{3} / \mathrm{mm}^{3}$ & $11.0(9.3-13.6)$ & $9.3(7.8-11.4)$ & $10.1(8.6-12.6)$ & $<0.001$ \\
\hline CK peak, IU/L & 692.5(207.5-1639.5) & 473.5(159.3-1430.0) & $541.5(224.0-1828.5)$ & 0.397 \\
\hline Angiographic data & $N=84$ & $N=73$ & $N=67$ & \\
\hline Number diseased vessels & & & & 0.009 \\
\hline 0 & $1(1 \%)$ & $4(5 \%)$ & $2(3 \%)$ & \\
\hline 1 & $41(49 \%)$ & $27(37 \%)$ & $28(42 \%)$ & \\
\hline 2 & $34(40 \%)$ & $21(29 \%)$ & $16(24 \%)$ & \\
\hline 3 or LM & $8(9 \%)$ & $21(29 \%)$ & $21(31 \%)$ & \\
\hline
\end{tabular}

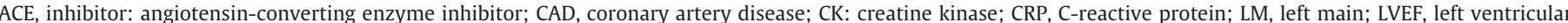

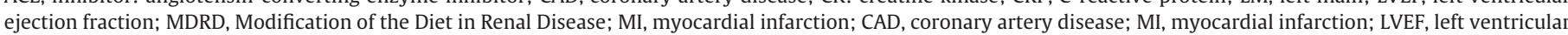
ejection fraction; STEMI: ST segment elevation MI.

Circulating leukocytes, which are exposed to the systemic environment including risk factors, are directly involved in low-grade inflammation related to atherosclerosis, may be useful to improve phenotype of the patient. Furthermore, using new techniques it is possible to explore the leukocyte transriptome to assess the systemic consequences of CV risk factors, including smoking [14]. A recent large community-based cohort study demonstrated the clinical correlates of gene expression, and the results were consistent with the hypothesis that leukocyte inflammatory transcripts play a role in the pathogenesis of CAD and its risk factors [15].

\section{1. c-Fos and atherosclerosis}

The $c$-Fos gene is rapidly activated in the presence of different stimuli, resulting in a mitogenic effect, which has been shown in many proliferative processes, particularly during embryonic development and in some types of tumor $[16,17]$. The $c$-Fos gene is also known to regulate the growth, differentiation, and migration of a variety of vascular and non-vascular cells, and to regulate matrix degradation [18]. A panel of genes, including $c$-Fos, that were differentially expressed in microarray analysis, was clearly able to identify acute ischemic stroke patients from healthy subjects [19]. Immunohistological analyses have shown that c-Fos expression characterizes the initial phases of the destabilization of unstable atherosclerotic carotid plaques [20]. Moreover, c-Fos is highly expressed and collocated with macrophage CD14+ immunoreactivity in human coronary artery plaque, suggesting its potential involvement in pathogenesis [8]. c-Fos overexpression may also promote early atherosclerotic coronary lesions by causing the proliferation of smooth muscle cells of the media [21]. Interestingly, an association between early atherosclerosis and c-Fos transcription was found in arterial biopsies of prenatal infants and infants with mothers who smoked [22].

\section{2. c-Fos and smoking}

Our study, which was supported by multivariate adjustments that included many demographic and biological variables, showed for the first time that in CAD patients, $c$-Fos transcript levels were associated with smoking status, independently of classical markers of inflammation, such as CRP or WBC. These findings are consistent with data from the literature, which show that conventional markers of inflammation are not significantly higher in smokers than in non-smokers. In a large contemporary randomized prospective clinical trial of current smokers, smoking intensity was not associated with CRP levels, and smoking cessation did not reduce CRP levels [23]. The effect of smoking reported in the present study seems specifically targeted to the blood 
Table 2

Univariate predictors of $c$-Fos transcript levels (AU).

\begin{tabular}{|c|c|c|c|c|c|}
\hline & & Median & IQR & $r$ & $p$ \\
\hline \multicolumn{6}{|l|}{ Risk factors } \\
\hline Age & & & & +0.005 & 0.943 \\
\hline \multirow[t]{2}{*}{ Female } & Yes & 0.900 & $0.837-0.969$ & & 0.703 \\
\hline & No & 0.900 & $0.838-0.980$ & & \\
\hline \multirow[t]{2}{*}{ Hypertension } & Yes & 0.891 & $0.827-0.957$ & & 0.188 \\
\hline & No & 0.906 & $0.848-0.993$ & & \\
\hline \multirow[t]{2}{*}{ Obesity } & Yes & 0.900 & $0.584-0.970$ & & 0.980 \\
\hline & No & 0.897 & $0.835-0.980$ & & \\
\hline \multirow[t]{2}{*}{ Diabetes } & Yes & 0.909 & $0.840-0.970$ & & 0.993 \\
\hline & No & 0.891 & $0.837-0.981$ & & \\
\hline \multirow[t]{2}{*}{ Hypercholesterolemia } & Yes & 0.925 & $0.839-0.987$ & & 0.274 \\
\hline & No & 0.891 & $0.836-0.963$ & & \\
\hline \multirow[t]{2}{*}{ Family history of CAD } & Yes & 0.891 & $0.805-0.953$ & & 0.070 \\
\hline & No & 0.907 & $0.848-0.990$ & & \\
\hline \multirow[t]{2}{*}{ Prior MI } & Yes & 0.891 & $0.824-0.925$ & & 0.064 \\
\hline & No & 0.908 & $0.840-0.987$ & & \\
\hline \multicolumn{6}{|l|}{ Clinical data on admission } \\
\hline LVEF & & & & +0.007 & 0.919 \\
\hline Systolic blood pressure & & & & -0.083 & 0.205 \\
\hline Diastolic blood pressure & & & & -0.093 & 0.158 \\
\hline Heart rate & & & & 0.073 & 0.268 \\
\hline \multirow[t]{2}{*}{ Anterior wall } & Yes & 0.910 & $0.847-0.984$ & & 0.326 \\
\hline & No & 0.834 & $0.691-0.975$ & & \\
\hline \multicolumn{6}{|l|}{ Chronic treatments } \\
\hline \multirow[t]{2}{*}{ ACE inhibitor } & Yes & 0.886 & $0.823-0.943$ & & 0.174 \\
\hline & No & 0.908 & $0.845-0.982$ & & \\
\hline \multirow[t]{2}{*}{ Statin } & Yes & 0.909 & $0.848-0.973$ & & 0.796 \\
\hline & No & 0.895 & $0.836-0.979$ & & \\
\hline \multirow[t]{2}{*}{ Aspirin } & Yes & 0.900 & $0.855-0.946$ & & 0.775 \\
\hline & No & 0.901 & $0.836-0.991$ & & \\
\hline \multirow[t]{2}{*}{ Betablocker } & Yes & 0.893 & $0.814-0.935$ & & 0.067 \\
\hline & No & 0.909 & $0.846-0.989$ & & \\
\hline \multirow[t]{2}{*}{ Fibrate } & Yes & 0.979 & $0.821-1.055$ & & 0.226 \\
\hline & No & 0.897 & $0.838-0.975$ & & \\
\hline \multirow[t]{2}{*}{ Oral antidiabetic } & Yes & 0.900 & $0.837-0.978$ & & 0.397 \\
\hline & No & 0.925 & $0.838-0.979$ & & \\
\hline \multicolumn{6}{|l|}{ Biological data } \\
\hline Onset to sampling & & & & +0.084 & 0.213 \\
\hline Glucose & & & & +0.003 & 0.969 \\
\hline HbA1c & & & & +0.106 & 0.125 \\
\hline MDRD & & & & +0.067 & 0.302 \\
\hline CRP & & & & +0.012 & 0.852 \\
\hline Homocysteine & & & & +0.098 & 0.168 \\
\hline HDL-cholest & & & & +0.066 & 0.329 \\
\hline Triglycerides & & & & -0.015 & 0.829 \\
\hline LDL-cholest & & & & -0.004 & 0.949 \\
\hline Total-cholest & & & & -0.004 & 0.952 \\
\hline CK peak & & & & +0.009 & 0.895 \\
\hline NT-proBNP & & & & +0.038 & 0.568 \\
\hline Leukocyte count & & & & -0.019 & 0.711 \\
\hline Angiographic data & & & & & \\
\hline Number of diseased vessels & & & & & 0.465 \\
\hline & 0 & 0.848 & $0.811-0.908$ & & \\
\hline & 1 & 0.889 & $0.825-0.979$ & & \\
\hline & 2 & 0.909 & $0.856-0.991$ & & \\
\hline & 3 or LM & 0.899 & $0.837-0.958$ & & \\
\hline
\end{tabular}

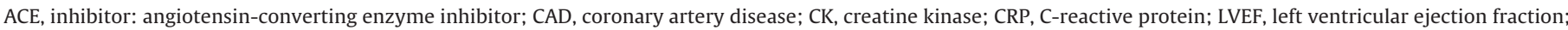
MDRD, Modification of the Diet in Renal Disease; MI, myocardial infarction.

leukocyte transcriptome such as c-Fos. The results of the multivariate analysis also showed that the lower use of treatments such as betablocker or ACEi does not explain, or only partly explains the elevated levels of FOS in the CS group. There was a gradual increase in expression according to smoking status. Past smokers had a significantly higher level of c-Fos transcript than never smokers but a lower level than that in current smokers. This reversible relationship is consistent with the known reversible $\mathrm{CV}$ risk associated with tobacco use and suggests that the $c$ Fos transcript level could be considered a cumulative biomarker of exposure to cigarette smoke. The underlying mechanism of the high levels of $c$-Fos induced by smoking remains unclear. Cigarette smoke is a complex mixture of $>4000$ chemical substances that are distributed in particulate and gaseous phases. It has been difficult to identify the pathophysiologically relevant components of cigarette smoke. Nicotine combustion products, the major psychoactive component in tobacco smoke, could promote the activation of genes such as c-Fos, further promoting other genes involved in smooth muscle cell proliferation and/or apoptosis. Nicotine-induced c-Fos immunostaining has been reported in rat brain [24], and the co-administration of nicotine and Ang II induced DNA synthesis and c-Fos expression in adult rat aortic VSMC and adventitial fibroblasts [11]. Whether smoking is also associated with increased expression of pro-apoptosis genes, such as $\mathrm{p} 53$ or $\mathrm{p} 16 \mathrm{Ink} 4 \mathrm{a}$, in such high risk patients remains to be determined. 


\subsection{Study limitation}

This study suffers from the usual limitation of non-randomized studies in that it determined correlations rather than causal relationships. Moreover, no assessment of smoking intensity or carbon monoxide levels was performed. However, the main strengths of this work are the use of a prospective design and a comprehensive analysis of a large number of confounding variables to analyze factors that affect the circulating leukocyte transcriptome. Moreover, we did not assess either smoking intensity or biological markers of exposure to tobacco smoke. Because biomarkers of exposure to cigarette smoke, such as cotinine levels, are highly specific to tobacco products, previous epidemiologic studies have used biomarkers of exposure to cigarette smoke to verify the accuracy of self-reported smoking. Quantitative biomarker data provide significantly more detailed and objective information on tobacco smoke exposure than does smoking history. It has been shown that self-reported smoking history markedly underestimates exposure to cigarette smoke in high risk patients [25]. A strong association between systemic inflammatory response and cotinine levels to measure second-hand exposure [26], or active smoking [27] has also been reported. However, both smokeless tobacco and nicotine replacement therapy, which may have been used by the high risk patients of our study, can raise nicotine levels, potentially confounding the interpretation of markers of exposure to cigarette smoke. Moreover, there is no gold standard to measure exposure to cigarette smoke, and, historically, self-reported smoking remains the standard for comparison in the real world setting.

\section{Conclusion}

The results of our investigation offer insights into cigarettesmoke related pathophysiologic processes by determining for the first time that smoking status is significantly associated with elevated levels of circulating c-Fos transcripts. If these findings are confirmed in larger prospective studies, our work suggests that the level of c-Fos transcripts could be considered as a biomarker of cumulative exposure to tobacco smoke. As c-Fos has been implicated in the progression and severity of atherosclerosis, it could be considered a potential pathway for tobacco toxicity in coronary artery disease.

\section{Conflict of interest}

No conflicts of interest to disclose that could inappropriately influence the present work.

\section{Acknowledgements}

This work was supported by the University Hospital of Dijon, the Association de Cardiologie de Bourgogne and by grants from the Union Régionale des Caisses d'Assurance Maladie de Bourgogne (URCAM), Agence Régionale d'Hospitalisation (ARH) et Agence Régionale de Santé (ARS) de Bourgogne, Conseil Régional de Bourgogne and Fédération Française de Cardiologie (FFC). We wish to thank Jean-Claude Beer, Edith Fusier and Martine
Jeanningros for research assistance and Philip Bastable for English assistance.

\section{References}

[1] Teo KK, et al. Tobacco use and risk of myocardial infarction in 52 countries in the INTERHEART study: a case-control study. Lancet 2006;368(9536):647-58.

[2] Mackay J, Ericksen M. The Tobacco atlas. Geneva: World Health Organization; 2002.

[3] Nishi K, et al. Oxidized LDL in carotid plaques and plasma associates with plaque instability. Arterioscler Thromb Vasc Biol 2002;22(10):1649-54.

[4] Schindler TH, et al. Coronary vasoregulation in patients with various risk factors in response to cold pressor testing: contrasting myocardial blood flow responses to short- and long-term vitamin C administration. J Am Coll Cardiol 2003;42(5):814-22.

[5] Craig WY, Palomaki GE, Haddow JE. Cigarette smoking and serum lipid and lipoprotein concentrations: an analysis of published data. BMJ 1989;298(6676):784-8.

[6] Calapai G, et al. A cross-sectional investigation of biomarkers of risk after a decade of smoking. Inhal Toxicol 2009;21(13):1138-43.

[7] Yanbaeva DG, et al. Systemic effects of smoking. Chest 2007;131(5):1557-66.

[8] Patino WD, et al. Circulating transcriptome reveals markers of atherosclerosis. Proc Natl Acad Sci USA 2005;102(9):3423-8.

[9] Kang JG, et al. FOS expression in blood as a LDL-independent marker of statin treatment. Atherosclerosis 2010;212(2):567-70.

[10] Ning W, et al. Comprehensive gene expression profiles reveal pathways related to the pathogenesis of chronic obstructive pulmonary disease. Proc Natl Acad Sci USA 2004;101(41):14895-900.

[11] Li JM, et al. Nicotine enhances angiotensin II-induced mitogenic response in vascular smooth muscle cells and fibroblasts. Arterioscler Thromb Vasc Biol 2004;24(1):80-4.

[12] Warnick GR, et al. Estimating low-density lipoprotein cholesterol by the Friedewald equation is adequate for classifying patients on the basis of nationally recommended cutpoints. Clin Chem 1990;36(1):15-9.

[13] Levey AS, et al. A more accurate method to estimate glomerular filtration rate from serum creatinine: a new prediction equation. Modification of Diet in Renal Disease Study Group. Ann Intern Med 1999;130(6):461-70.

[14] Ardigo D, Gaillard CA, Braam B. Application of leukocyte transcriptomes to assess systemic consequences of risk factors for cardiovascular disease. Clin Chem Lab Med 2007;45(9):1109-20.

[15] Freedman JE, et al. Relation of platelet and leukocyte inflammatory transcripts to body mass index in the Framingham heart study. Circulation 2010;122(2):119-29.

[16] Dony C, Gruss P. Proto-oncogene c-fos expression in growth regions of fetal bone and mesodermal web tissue. Nature 1987;328(6132):711-4.

[17] Joseph P, et al. Cadmium-induced cell transformation and tumorigenesis are associated with transcriptional activation of c-fos, c-jun, and c-myc protooncogenes: role of cellular calcium and reactive oxygen species. Toxicol Sci 2001;61(2):295-303.

[18] Goetze S, et al. TNFalpha induces expression of transcription factors c-fos: Egr1 , and Ets-1 in vascular lesions through extracellular signal-regulated kinases $1 / 2$. Atherosclerosis 2001;159(1):93-101.

[19] Moore DF, et al. Using peripheral blood mononuclear cells to determine a gene expression profile of acute ischemic stroke: a pilot investigation. Circulation 2005;111(2):212-21.

[20] Lavezzi AM, et al. Expression of c-fos: p53 and PCNA in the unstable atherosclerotic carotid plaque. Int J Cardiol 2003;92(1):59-63.

[21] Milei J, et al. Perinatal and infant early atherosclerotic coronary lesions. Can J Cardiol 2008;24(2):137-41.

[22] Matturri L, et al. Intimal preatherosclerotic thickening of the coronary arteries in human fetuses of smoker mothers. J Thromb Haemost 2003;1(10):2234-8.

[23] Asthana A, et al. Effects of smoking intensity and cessation on inflammatory markers in a large cohort of active smokers. Am Heart J 2010;160(3):458-63.

[24] Seppa T, et al. Induction of Fos-immunostaining by nicotine and nicotinic receptor antagonists in rat brain. Neuropharmacology 2001:41(4):486-95.

[25] Hsieh SJ, et al. Biomarkers increase detection of active smoking and secondhand smoke exposure in critically ill patients. Crit Care Med 2011;39(1):40-5.

[26] Jefferies BJ, et al. Secondhand smoke (SHS) exposure is associated with circulating markers of inflammation and endothelial function in adult men and women. Atherosclerosis 2010;208(2):550-6.

[27] Bazzano LA, et al. Relationship between cigarette smoking and novel risk factors for cardiovascular disease in the United States. Ann Intern Med 2003;138(11):891-7. 